\title{
LES GUIDES CYLINDRIQUES SUPRACONDUCTEURS EN HYPERFRÉQUENCES
}

\author{
G. ZEPP \\ Laboratoire de Physique Théorique, Faculté des Sciences, Clermont-Ferrand
}

(Reçu le 30 novembre 1970)

\begin{abstract}
Résumé. - On étudie le comportement d'un guide cylindrique supraconducteur, constitué par un matériau de longueur de cohérence $\xi_{0}$ élevée, en présence d'une onde électromagnétique hyperfréquence. On détermine en particulier les variations du coefficient d'atténuation, du déphasage ainsi que celles de la profondeur de pénétration des champs en fonction du degré d'impureté du matériau utilisé.
\end{abstract}

Abstract. - The behaviour of a cylindrical superconducting guide (with a large coherence length $\xi_{0}$ ) at high frequencies has been studied. The variations of attenuation coefficient and penetration depth of the fields with the amount of impurities are determined.

I. Introduction. - Comme dans une précédente étude [1] concernant les supraconducteurs ayant une longueur de cohérence $\xi_{0}$ élevée (en présence d'un champ électromagnétique) nous considérons que la densité de courant électronique en un point intérieur au matériau supraconducteur peut être mise sous la forme :

$$
\mathbf{J}=\mathbf{J}_{\boldsymbol{N}}+\mathbf{J}_{S}
$$

où $\mathbf{J}_{N}$ est la densité de courant associée aux électrons non appariés et $\mathbf{J}_{S}$ celle relative aux paires d'électrons.

Pour un matériau pur (le libre parcours moyen $l$ a alors une valeur élevée) on peut écrire :

$$
\mathbf{J}_{N}(\mathbf{r}, t)=\frac{3 \sigma}{4 \pi l} \int_{V} \mathbf{R} \frac{\hat{\mathbf{E}}\left(\mathbf{r}^{\prime}, t\right) \cdot \mathbf{R}}{R^{4}} \mathrm{e}^{-R / l} d \mathbf{r}^{\prime}
$$

avec $\mathbf{R}=\mathbf{r}^{\prime}-\mathbf{r}$ où $\mathbf{r}$ définit la position du point où l'on calcule $\mathbf{J}_{N}$ et $\mathbf{r}^{\prime}$ la position d'un point quelconque du volume $V$ constitué par le matériau. La conductivité effective $\sigma$ des électrons non appariés dépend de divers paramètres, en particulier de la fréquence du champ et de la température.

Dans le cas d'un matériau très jmpur le libre parcours moyen $l$ devient très faible. L'expression précédente donnant $\mathbf{J}_{N}$ peut alors s'écrire :

$$
\mathbf{J}_{N} \simeq \sigma \mathbf{E} \text {. }
$$

L'expression de $\mathbf{J}_{S}$ est donnée par :

$$
\mathbf{J}_{S}=-\frac{3}{4 \pi} \frac{1}{\mu \lambda_{L}^{2} \xi_{0}} \int_{V} \mathbf{R} \frac{\mathbf{A}\left(r^{\prime}, t\right) \cdot \mathbf{R}}{R^{4}} \mathrm{e}^{-R / \xi} d \mathbf{r}^{\prime}
$$

où $\xi$ est relié à la longueur de cohérence $\xi_{0}$ et au libre parcours moyen $l$ par la relation :

$$
\frac{1}{\xi}=\frac{1}{\xi_{0}}+\frac{k}{l}
$$

$k$ étant sans dimension et voisin de l'unité.
Si $\xi$ est faible on peut écrire :

$$
\mathbf{J}_{S} \simeq-\frac{1}{\mu \lambda_{L}^{2}} \frac{\xi}{\xi_{0}} \mathbf{A} \quad \text { (loi locale) } .
$$

Comme $\xi_{0}$ est supposé grand, une valeur faible pour $\xi$ ne peut être obtenue que si $l$ est lui-même faible ce qui correspond à un matériau impur. On a d'ailleurs dans ce cas $\xi \simeq l$.

Pour un matériau pur on doit donc conserver pour $\mathbf{J}_{S}$ la forme intégrale (loi non locale).

D'autre part nous admettons que les électrons subissent une réflexion diffuse à la surface limitant le supraconducteur (cas le plus courant en pratique).

On peut alors déterminer la valeur de l'impédance de surface $Z_{S}$. Etudions le cas d'une surface plane (les résultats nous seront utiles dans la suite de l'exposé).

Si le matériau est très impur les lois locales (2) et (4) sont alors applicables. En appelant $Z_{S}^{\prime}$ la valeur de l'impédance de surface dans ce cas, on obtient ( $\omega$ étant la pulsation du champ) :

$$
Z_{S}^{\prime}=i \mu \omega\left(\frac{1}{\lambda_{L}^{2}} \frac{\xi}{\check{\zeta}_{0}}+i \mu \omega \sigma\right)^{-1 / 2}
$$

Dans le cas d'un matériau pur ou presque pur, le calcul, plus difficile car il conduit à la résolution d'une équation intégrale du type Wiener-Hopf, donne [1] :

$$
\begin{aligned}
Z_{S} \simeq \frac{\sqrt{3}}{2} i \mu \omega g_{1}^{-1 / 3} \times & \\
& \times\left(1-\frac{\sqrt{3}}{2 \pi} \frac{g_{2}}{g_{1}^{4 / 3}}\left(1-\log \frac{g_{2}}{g_{1}^{4 / 3}}\right)\right)
\end{aligned}
$$

avec $\left|g_{2}\right| \ll\left|g_{1}^{4 / 3}\right|$, les expressions de $g_{1}$ et $g_{2}$ étant : 


$$
g_{1}=\frac{3 \pi}{4}\left(\frac{1}{\xi_{0} \lambda_{L}^{2}}+i \frac{\mu \omega \sigma}{l}\right)
$$

et

$$
g_{2}=-\frac{3}{2}\left(\frac{1}{\xi_{0}} \frac{\xi \lambda_{L}^{2}}{i}+i \frac{\mu \omega \sigma}{l^{2}}\right) .
$$

Une valeur plus approchée est :

$$
Z_{S} \simeq \frac{\sqrt{3}}{2} i \mu \omega g_{1}^{-1 / 3}
$$

On peut déduire de l'expression de $Z_{S}$ la valeur de la résistance de surface $R_{S}$.

Pour un matériau pur on obtient en utilisant (6) :

$$
\begin{aligned}
R_{S}=\frac{\sqrt{3}}{2 \pi}\left(\frac{4 \xi_{0} \lambda_{L}^{2}}{3 \pi}\right)^{1 / 3} \times & \\
& \times \frac{\mu \omega \sin \left(\frac{1}{3} \operatorname{Arctg} \frac{\mu \omega \sigma \xi_{0} \lambda_{L}^{2}}{l}\right)}{\left(1+\left(\frac{\mu \omega \sigma \xi_{0} \lambda_{L}^{2}}{l}\right)^{2}\right)^{1 / 6}}\{\}
\end{aligned}
$$

où \{\} représente la correction à apporter à l'expression de $R_{S}$ que l'on déduirait de (7).

Dans le cas général le terme correctif de (8) a une expression assez complexe. Dans le cas particulier important en pratique où :

$$
\frac{\mu \omega \sigma \xi_{0} \lambda_{L}^{2}}{l} \ll 1
$$

donc également

$$
\frac{\mu \omega \sigma \xi_{0} \xi \lambda_{L}^{2}}{i^{2}} \ll 1
$$

on peut écrire pour un matériau très pur $(l \gg \xi)$

$$
\begin{aligned}
\{\} \simeq 1+\frac{\sqrt{3}}{\pi^{2}} & \left(\frac{4}{3 \pi} \frac{\lambda_{L}^{2}}{\xi_{0}^{2}}\right)^{1 / 3} \times \\
& \times\left(1-5 \log \frac{2}{\pi}\left(\frac{4}{3 \pi} \frac{\lambda_{L}^{2}}{\xi_{0}^{2}}\right)^{1 / 3}\right) .
\end{aligned}
$$

Dans le cas de l'aluminium par exemple, si $T \ll T_{\text {c }}$ ( $T_{\mathrm{c}}$ température critique) on a $\lambda_{L} \simeq 165 \AA$ et $\xi_{0}=16000 \AA$. Le rapport $\lambda_{L} / \xi_{0}$ est donc très proche de $10^{-2}$. La correction est alors voisine de $12 \%$, donc non négligeable.

D'autre part dans l'état supraconducteur on peut écrire : $\sigma=f_{N} \sigma_{N}$ où $f_{N}$ est la fraction des électrons qui sont non appariés et $\sigma_{N}$ la valeur de $\sigma$ pour $T=T_{\mathrm{c}}\left(f_{N}=1\right) . f_{N}$ dépend en particulier de la pulsation $\omega$ et de la température $T$, mais si la fréquence n'est pas trop élevée on peut écrire :

$$
f_{N} \simeq\left(\frac{T}{T_{\mathrm{c}}}\right)^{4} \quad \text { et } \quad \lambda_{L}\left(\frac{T}{T_{\mathrm{c}}}\right) \simeq \frac{\lambda_{L}(0)}{\left(1-\left(T / T_{\mathrm{c}}\right)^{4}\right)^{1 / 2}} .
$$

Dans l'approximation précédente, on admet donc que, pour $T=0, f_{N}=0$, c'est-à-dire que tous les électrons sont supraconducteurs. Or ceci n'est vrai que si $\hbar \omega \ll \Delta(0), \Delta(0)$ étant la largeur de la bande interdite à $0 \mathrm{o}$. Pour l'aluminium, la valeur critique de $\omega$ est voisine de $5 \times 10^{11} \mathrm{~s}^{-1}$.

En supposant que les relations (10) soient applicables, nous allons comparer les valeurs théoriques et expérimentales du rapport $r=R_{S} / R_{S N}\left(R_{S N}\right.$ est la résistance de surface dans l'état normal pour $T=T_{\mathrm{c}}$ ) pour $T$ variant de 0 à $T_{\mathrm{c}}$, la fréquence étant fixée.

En vue de la suite de l'exposé, la fréquence sera choisie dans la gamme des hyperfréquences habituellement utilisées en propagation guidée.

En utilisant les diagrammes expérimentaux obtenus par Biondi et Garfunkel pour l'aluminium [2] nous prenons par exemple $\omega=2,56 \times 10^{11} \mathrm{~s}^{-1}$ (fréquence $4,08 \times 10^{10} \mathrm{~Hz}$ ).

La figure 1 permet la comparaison. On voit sur le graphique que l'accord est très satisfaisant. Le terme

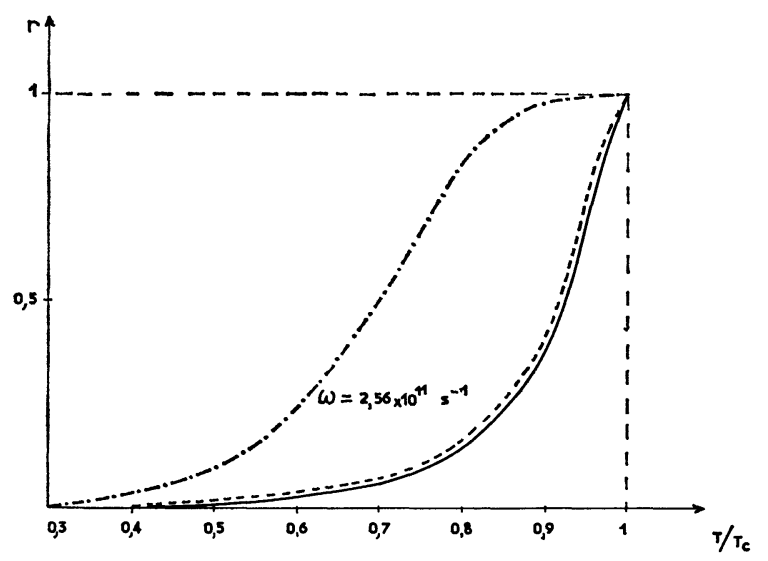

FIG. 1. - La courbe - - - correspond aux valeurs expérimentales. La courbe - correspond aux valeurs théoriques (non locales).

correctif est important puisqu'il est de l'ordre de plus de $15 \%$ pour certaines valeurs de $T / T_{\mathrm{c}}$. Il n'est négligeable qu'au voisinage très immédiat de $T_{\mathrm{c}}$. A noter que nous avons supposé la matériau très pur. En présence d'impuretés, la correction à apporter peut être supérieure. Par ailleurs nous avons tracé sur la figure 1 la courbe donnant $r\left(T / T_{\mathrm{c}}\right)$ en supposant qu'une théorie purement locale soit applicable pour un matériau pur (la valeur des paramètres est la même). On voit que cette théorie n'est plus satisfaisante dans le cas étudié (aluminium).

II. Propagation d'une onde plane électromagnétique dans un guide cylindrique supraconducteur [3]. Considérons une onde progressive se propageant dans un guide cylindrique d'axe $\mathrm{Oz}$ et de rayon $a$. En utilisant un système de coordonnées cylindriques $(\rho, \varphi, z)$, les composantes des champs constituant l'onde sont de la forme $f(\rho, \varphi) \mathrm{e}^{i(\omega t-\gamma z)}$. On étudie ici plus en détail les modes $\mathrm{TM}_{01}$ et $\mathrm{TE}_{01}$. On a alors : $f(\rho, \varphi)=f(\rho)$.

1 er CAS. Mode $\mathrm{TM}_{01}\left(\mathrm{H}_{\mathrm{z}}=0\right)$. - En présence d'impuretés le libre parcours moyen $l$ des électrons 
à l'intérieur du supraconducteur est faible. D'après ce que nous avons vu précédemment concernant les supraconducteurs de longueur de cohérence $\xi_{0}$ élevée, une formulation de type local pour les densités de courants normaux et supraconducteurs est dans ce cas possible.

Nous pouvons donc utiliser les relations (2) et (4).

Les équations de Maxwell permettent alors de déterminer les diverses composantes des champs $\mathbf{E}$ et $\mathbf{H}$ (à l'intérieur du guide et dans le métal).

Dans le vide, on obtient (à une constante multiplicative près) :

$$
\begin{gathered}
E_{\rho}=i \gamma \chi J_{1}(\chi \rho) \mathrm{e}^{i(\omega t-\gamma z)}, E_{\varphi}=0 \\
E_{z}=\chi^{2} J_{0}(\chi \rho) \mathrm{e}^{i(\omega t-\gamma z)} \\
H_{\rho}=0, H_{\varphi}=i k \chi \sqrt{\frac{\varepsilon_{0}}{\mu}} J_{1}(\chi \rho) \mathrm{e}^{i(\omega t-\gamma z)}, H_{z}=0
\end{gathered}
$$

avec $\chi^{2}=k^{2}-\gamma^{2} \quad$ et $\quad k^{2}=\mu \varepsilon_{0} \omega^{2}$.

Dans le métal supraconducteur :

$$
\begin{gathered}
E_{\rho}=E_{0} i \gamma \chi^{\prime} H_{1}^{(2)}\left(\chi^{\prime} \rho\right) \mathrm{e}^{i(\omega t-\gamma z)} \\
E_{\varphi}=0, E_{z}=E_{0} \chi^{\prime 2} H_{0}^{(2)}\left(\chi^{\prime} \rho\right) \mathrm{e}^{i(\omega t-\gamma z)} \\
H_{\rho}=0, H_{\varphi}=E_{0} i k^{\prime} \chi^{\prime} \sqrt{\frac{\varepsilon}{\mu}} H_{1}^{(2)}\left(\chi^{\prime} \rho\right) \mathrm{e}^{i(\omega t-\gamma z)} \\
H_{z}=0
\end{gathered}
$$

où

$$
k^{\prime 2}=\mu \varepsilon \omega^{2} \quad \chi^{\prime 2}=k^{\prime 2}-\gamma^{2}
$$

et

$$
\varepsilon=\varepsilon_{0}\left(1-\frac{\xi}{\mu \varepsilon_{0} \omega^{2} \xi_{0} \lambda_{L}^{2}}-\frac{i \sigma}{\varepsilon_{0} \omega}\right) .
$$

La fonction $H_{n}^{(2)}$ ( $n$ entier) est la fonction de Hankel :

$$
H_{n}^{(2)}=J_{n}-i \gamma_{n} \quad \text { (notations usuelles). }
$$

En écrivant la continuité de $H_{\varphi}$ et $E_{z}$ pour $\rho=a$ on obtient :

$$
\left.\frac{E_{z}}{H_{\varphi}}\right|_{\rho=a}=\frac{\chi J_{0}(\chi a)}{i \varepsilon_{0} \omega J_{1}(\chi a)}=\frac{\chi^{\prime} H_{0}^{(2)}\left(\chi^{\prime} a\right)}{i \varepsilon \omega H_{1}^{(2)}\left(\chi^{\prime} a\right)} .
$$

Par ailleurs, on peut écrire :

$$
\chi^{\prime 2}=\chi^{2}-\frac{\xi}{\dot{\zeta}_{0} \lambda_{L}^{2}}-i \mu \omega \sigma
$$

$\chi$ est voisin de $\chi_{0}$ tel que $\chi_{0}^{2}=k^{2}-\gamma_{0}^{2}, \gamma_{0}$ étant la constante de propagation correspondant à une conductivité infinie.

On a :

$$
\chi_{0}=\frac{2,4048}{a}
$$

2,4048 étant la première racine de $J_{0}(x)=0$.
Dans ces conditions $\left|\chi^{\prime} a\right| \gg 1$ et le rapport

$$
\frac{H_{0}^{(2)}\left(\chi^{\prime} a\right)}{H_{1}^{(2)}\left(\chi^{\prime} a\right)} \simeq-i
$$

valeur qui est indépendante du rayon $a$ du cylindre. (Pour établir ce résultat on a utilisé les formes asymptotiques de $H_{n}^{(2)}$.)

On en déduit que :

$$
\frac{\chi J_{0}(\chi a)}{i \varepsilon_{0} \omega J_{1}(\chi a)} \simeq-Z_{S}^{\prime}
$$

$Z_{S}^{\prime}$ étant l'impédance de surface d'un supraconducteur semi-infini dont la surface est plane. Dans le cadre de la théorie locale l'impédance de surface est donnée par (5) que l'on peut encore écrire :

$$
\begin{aligned}
\left.Z_{S}^{\prime}=\frac{i \mu \omega}{\left(\left(\frac{\xi}{\xi_{0} \lambda_{L}^{2}}\right)^{2}+\right.}+(\mu \omega \sigma)^{2}\right)^{1 / 4} \times \\
\quad \times\left(\cos \left(\frac{1}{2} \operatorname{Arctg} \frac{\mu \omega \sigma \lambda_{L}^{2} \xi_{0}}{\xi}\right)\right. \\
\left.-i \sin \left(\frac{1}{2} \operatorname{Arctg} \frac{\mu \omega \sigma \lambda_{L}^{2} \xi_{0}}{\xi}\right)\right)
\end{aligned}
$$

avec

$$
\operatorname{Arctg}\left(\frac{\mu \omega \sigma \lambda_{L}^{2} \xi_{0}}{\xi}\right) \quad \text { compris entre } 0 \text { et } \frac{\pi}{2} .
$$

Posons : $\gamma=\gamma_{0}+\delta^{\prime} \gamma_{0}-i \alpha^{\prime}$ où $\alpha^{\prime}$ est le coefficient d'atténuation de l'onde.

En tenant compte du fait que $\chi$ est voisin de $\chi_{0}$ et en admettant que $\gamma_{0}$ est assez grand par rapport à $\delta^{\prime} \gamma_{0}$ et $\alpha^{\prime}$ on peut déterminer les valeurs de $\delta^{\prime} \gamma_{0}$ et $\alpha^{\prime}$.

Comme la théorie locale n'est applicable que si $l$ est faible, on a alors $\xi \simeq l$. On obtient finalement :

$$
\delta^{\prime} \gamma_{0} \simeq \frac{\mu \varepsilon_{0} \omega^{2}}{\gamma_{0} a} \frac{\cos \left(\frac{1}{2} \operatorname{Arctg} \mu \omega \sigma \lambda_{L}^{2} \frac{\xi_{0}}{l}\right)}{\left(\left(\frac{1}{\lambda_{L}^{2}} \frac{l}{\xi_{0}}\right)^{2}+(\mu \omega \sigma)^{2}\right)^{1 / 4}}
$$

avec

$$
\gamma_{0} \simeq\left(\mu \varepsilon_{0} \omega^{2}-\frac{(2,40)^{2}}{a^{2}}\right)^{1 / 2}
$$

$\delta^{\prime} \gamma_{0}$ permet de déterminer la diminution de la vitesse de propagation par rapport au cas où la conductivité est supposée infiniment grande.

Les champs sont proportionnels à $\mathrm{e}^{-\alpha^{\prime} z}$ avec :

$$
\alpha^{\prime} \simeq \frac{\mu \varepsilon_{0} \omega^{2}}{\gamma_{0} a} \frac{\sin \left(\frac{1}{2} \operatorname{Arctg} \mu \omega \sigma \lambda_{L}^{2} \frac{\xi_{0}}{l}\right)}{\left(\left(\frac{1}{\lambda_{L}^{2}} \frac{l}{\xi}\right)^{2}+(\mu \omega \sigma)^{2}\right)^{1 / 4}} .
$$


A la transition $\lambda_{L}$ tend vers $+\infty$ et $\alpha^{\prime}$ tend vers l'expression classique du coefficient d'atténuation correspondant à un métal ordinaire $\left(\sigma=\sigma_{N}\right)$ :

$$
\alpha_{N} \simeq \frac{\mu \varepsilon_{0} \omega^{2}}{\gamma_{0} a} \frac{1}{\left(2 \mu \omega \sigma_{N}\right)^{1 / 2}} .
$$

Dans le cas où le matériau utilisé est pur, le libre parcours moyen $l$ est grand (on est à très basse température). On est donc amené à utiliser les lois non locales (1) et (3) pour $\mathbf{J}_{N}$ et $\mathbf{J}_{S}$ respectivement.

Nous supposerons que 1 'on a $1 / l \gg|\gamma|$. On peut alors en déduire que l'on peut écrire :

$$
\frac{\chi J_{0}(\chi a)}{i \varepsilon_{0} \omega J_{1}(\chi a)} \simeq-Z_{S}
$$

où $Z_{S}$ est la valeur de l'jmpédance de surface déduite de la formulation non locale précédente dans le cas d'une surface plane. Nous utilisons donc ici les expressions (6) ou (7) de $Z_{S}$ suivant le degré de précision recherché.

Pour simplifier, utilisons la forme (7) de $Z_{S}$. Posant : $\gamma=\gamma_{0}+\delta \gamma_{0}-i \alpha$ on peut en déduire par un calcul analogue à celui fait en théorie locale des expressions approchées pour $\delta \gamma_{0}$ et $\alpha$. On a :

$$
\begin{aligned}
\delta \gamma_{0} \simeq \frac{\sqrt{3}}{2}\left(\frac{4}{3 \pi}\right)^{1 / 3} & \frac{\mu \varepsilon_{0} \omega^{2}}{\gamma_{0} a} \times \\
& \times \frac{\cos \left(\frac{1}{3} \operatorname{Arctg} \mu \omega \sigma \lambda_{L}^{2} \frac{\xi_{0}}{l}\right)}{\left(\left(\frac{1}{\xi_{0} \lambda_{L}^{2}}\right)^{2}+\left(\frac{\mu \omega \sigma}{l}\right)^{2}\right)^{1 / 6}} .
\end{aligned}
$$

La valeur de $\gamma_{0}$ est toujours donnée par (12).

Le coefficient d'atténuation de l'onde est :

$$
\begin{aligned}
\alpha=\frac{\sqrt{3}}{2}\left(\frac{4}{3 \pi}\right)^{1 / 3} \frac{\mu \varepsilon_{0} \omega^{2}}{\gamma_{0} a} \times \\
\\
\times \frac{\sin \left(\frac{1}{3} \operatorname{Arctg} \mu \omega \sigma \lambda_{L}^{2} \frac{\xi_{0}}{l}\right)}{\left(\left(\frac{1}{\xi_{0} \lambda_{L}^{2}}\right)^{2}+\left(\frac{\mu \omega \sigma}{l}\right)^{2}\right)^{1 / 6}} .
\end{aligned}
$$

Les expressions (14) et (15) de $\delta \gamma_{0}$ et $\alpha$ respectivement sont à comparer avec celles $\{(11)$ et (13) $\}$ correspondant à la théorie locale.

$2^{\mathrm{e}}$ CAS. Mode $\mathrm{TE}_{01}\left(\mathrm{E}_{\mathrm{z}}=0\right)$. $-\mathrm{Si}$ les densités de courant normal et supraconducteur peuvent être décrites par des relations de type local (matériau très impur), les équations de Maxwell conduisent à écrire pour les différentes composantes des champs :

Dans le vide (à une constante multiplicative près) :

$$
\begin{gathered}
E_{\rho}=0 \quad E_{\varphi}=-i k \chi \sqrt{\frac{\mu}{\varepsilon_{0}}} J_{1}(\chi \rho) \mathrm{e}^{i(\omega t-\gamma z)} E_{z}=0 \\
H_{\rho}=i \gamma \chi J_{1}(\chi \rho) \mathrm{e}^{i(\omega t-\gamma z)} \quad H_{\varphi}=0 \\
H_{z}=\chi^{2} J_{0}(\chi \rho) \mathrm{e}^{i(\omega t-\gamma z)} .
\end{gathered}
$$

Dans le métal supraconducteur :

$$
\begin{gathered}
E_{\rho}=0 E_{\varphi}=-E_{0} i k^{\prime} \chi^{\prime} \sqrt{\frac{\mu}{\varepsilon}} H_{1}^{(2)}\left(\chi^{\prime} \rho\right) \mathrm{e}^{i(\omega t-\gamma z)} \\
E_{z}=0 \\
H_{\rho}=E_{0} i \gamma \chi^{\prime} H_{1}^{(2)}\left(\chi^{\prime} \rho\right) \mathrm{e}^{i(\omega t-\gamma z)} H_{\varphi}=0 \ldots \\
\ldots H_{z}=E_{0} \chi^{\prime 2} H_{0}^{(2)}\left(\chi^{\prime} \rho\right) \mathrm{e}^{i(\omega t-\gamma z)} .
\end{gathered}
$$

Les notations sont les mêmes que pour le mode $\mathrm{TM}_{01}$. Un raisonnement analogue à celui fait pour le mode $\mathrm{TM}_{01}$ montre que l'on peut écrire :

$$
\left.\frac{E_{\varphi}}{H_{z}}\right|_{\rho=a}=-\frac{i k \sqrt{\frac{\mu}{\varepsilon_{0}}} J_{1}(\chi a)}{\chi J_{0}(\chi a)} \simeq Z_{S}^{\prime}
$$

où $Z_{S}^{\prime}$ est l'impédance de surface d'un milieu plan semi-infini. Soit $\chi_{0}$ la valeur de $\chi$ correspondant à une conductivité infinie. $\chi_{0}$ est tel que $J_{1}\left(\chi_{0} a\right)=0$, soit $\chi_{0} a \simeq 3,83$, où 3,83 est la première racine non nulle de $J_{1}(x)=0$.

En posant comme dans l'étude du mode $\mathrm{TM}_{01}$ :

$$
\gamma=\gamma_{0}+\delta^{\prime} \gamma_{0}-i \alpha^{\prime}
$$

et en remarquant que $\chi$ est voisin de $\chi_{0}$ et que $\delta^{\prime} \gamma_{0}$ et $\alpha^{\prime}$ ont une valeur très inférieure à celle de $\gamma_{0}$, on déduit de l'expression de :

$$
Z_{S}^{\prime}=i \mu \omega\left(\frac{1}{\lambda_{L}^{2}} \frac{\xi}{\xi_{0}}+i \mu \omega \sigma\right)^{-1 / 2}
$$

les valeurs de $\delta^{\prime} \gamma_{0}$ et $\alpha^{\prime}$ :

$$
\delta^{\prime} \gamma_{0} \simeq \frac{\chi_{0}^{2}}{a \gamma_{0}} \frac{\cos \left(\frac{1}{2} \operatorname{Arctg} \mu \omega \sigma \lambda_{L}^{2} \frac{\xi_{0}}{\xi}\right)}{\left(\left(\frac{1}{\lambda_{L}^{2}} \frac{\xi}{\xi_{0}}\right)^{2}+(\mu \omega \sigma)^{2}\right)^{1 / 4}}
$$

avec dans ce cas :

$$
\gamma_{0}=\left(\mu \varepsilon_{0} \omega^{2}-\frac{(3,83)^{2}}{a^{2}}\right)^{1 / 2} .
$$

Il faut également rappeler que l'on a alors $\xi \simeq l$ ( $l$ faible). Le coefficient d'atténuation $\alpha^{\prime}$ a pour valeur :

$$
\alpha^{\prime} \simeq \frac{\chi_{0}^{2}}{a \gamma_{0}} \frac{\sin \left(\frac{1}{2} \operatorname{Arctg} \mu \omega \sigma \lambda_{L}^{2} \frac{\xi_{0}}{\xi}\right)}{\left(\left(\frac{1}{\lambda_{L}^{2}} \frac{\xi}{\xi_{0}}\right)^{2}+(\mu \omega \sigma)^{2}\right)^{1 / 4}}
$$

Si le matériau utilisé est pur ( $l$ est alors grand), on déduit de la valeur (7) de $Z_{S}$ (théorie non locale) l'expression de $\delta \gamma_{0}$ et du coefficient d'atténuation $\alpha$ (on suppose toujours $1 / l \gg|\gamma|$ et on a posé $\left.\gamma=\gamma_{0}+\delta \gamma_{0}-i \alpha\right)$ : 
$\delta \gamma_{0} \simeq \frac{\sqrt{3}}{2}\left(\frac{4}{3 \pi}\right)^{1 / 3} \frac{\chi_{0}^{2}}{a \gamma_{0}} \frac{\cos \left(\frac{1}{3} \operatorname{Arctg} \mu \omega \sigma \lambda_{L}^{2} \frac{\xi_{0}}{l}\right)}{\left(\left(\frac{1}{\lambda_{L}^{2}} \frac{1}{\xi_{0}}\right)^{2}+\left(\frac{\mu \omega \sigma}{l}\right)^{2}\right)^{1 / 6}}$

et

$$
\alpha \simeq \frac{\sqrt{3}}{2}\left(\frac{4}{3 \pi}\right)^{1 / 3} \frac{\chi_{0}^{2}}{a \gamma_{0}} \frac{\sin \left(\frac{1}{3} \operatorname{Arctg} \mu \omega \sigma \lambda_{L}^{2} \frac{\xi_{0}}{l}\right)}{\left(\left(\frac{1}{\lambda_{L}^{2}} \frac{1}{\xi_{0}}\right)^{2}+\left(\frac{\mu \omega \sigma}{l}\right)^{2}\right)^{1 / 6}} .
$$

Il convient de remarquer que les rapports $\alpha / \alpha^{\prime}$ et $\delta \gamma_{0} / \delta^{\prime} \gamma_{0}$ (pour un matériau donné, mais dont le degré de pureté varie) ont la même valeur que dans le cas du mode $\mathrm{TM}_{01}$ et que ces rapports sont indépendants du rayon $a$ du cylindre. On peut d'ailleurs noter que le rapport $\alpha / \alpha^{\prime}$ est le même que celui que l'on peut calculer dans le cas d'un guide plan parallèle $\{[1]\}$.

Supposons qu'une théorie non locale soit applicable pour déterminer $\alpha$ (matériau pur).

Nous calculons le rapport $\alpha_{S} / \alpha_{N}$ où $\alpha_{S}$ est le coefficient d'atténuation à une température $T<T_{\mathrm{c}}$ (état supraconducteur), et $\alpha_{N}$ le coefficient d'atténuation dans l'état normal pour $T=T_{\mathrm{c}}$. On a :

$\frac{\alpha_{S}}{\alpha_{N}} \simeq 2\left(\mu \omega \xi_{0} \lambda_{L}^{2} \frac{\sigma_{N}}{l}\right)^{1 / 3} \frac{\sin \left(\frac{1}{3} \operatorname{Arctg} \mu \omega \xi_{0} \lambda_{L}^{2} \frac{\sigma}{l}\right)}{\left(1+\left(\mu \omega \xi_{0} \lambda_{L}^{2} \frac{\sigma}{l}\right)^{2}\right)^{1 / 6}}$

$\operatorname{avec} \sigma=f_{N} \sigma_{N}$.

Pour obtenir cette expression on a négligé le terme correctif dans l'expression de $Z_{S}$ (formule (7)).

Il est facile de vérifier que même en tenant compte du terme correctif dans $Z_{S}$ (formule (6)) le rapport $\alpha_{S} / \alpha_{N}$ est identique à $r=R_{S} / R_{S N}$, rapport des résistances de surface à l'état supraconducteur $\left(T<T_{\mathrm{c}}\right)$ et à l'état normal $\left(T=T_{\mathrm{c}}\right)$.

La figure 1 représentant $r\left(T / T_{\mathrm{c}}\right)$ pour l'aluminium, permet donc d'étudier $\alpha_{S} / \alpha_{N}$ comme fonction de $T / T_{\mathrm{c}}$ (Fig. 2).

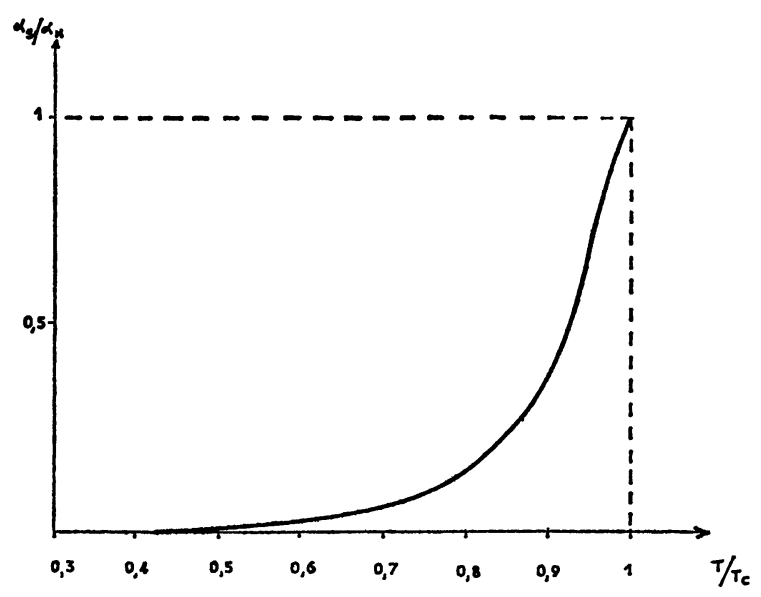

FIG. 2.
Dans l'exemple choisi on a $\omega=2,56 \times 10^{11} \mathrm{~s}^{-1}$. On pourrait, par exemple, choisir un guide de rayon $a \simeq 10^{-2} \mathrm{~m} ;$ pour le mode $\mathrm{TE}_{01}$ on aurait alors $\gamma_{0} \simeq 7,7 \times 10^{2} \mathrm{~m}^{-1}$.

Il faut rappeler que pour tracer les courbes des figures 1 ou 2 on a tenu compte du terme correctif dans l'expression de $Z_{S}$.

De même, si l'on veut une valeur plus précise pour $\delta \gamma_{0}$ il faut également utiliser (6).

On peut alors comparer la valeur de $\delta \gamma_{0}$ avec celle obtenue en admettant une théorie purement locale $\left(\delta^{\prime} \gamma_{0}\right)$ même dans le cas où le matériau est pur.

On étudie la variation du rapport $\delta \gamma_{0} / \delta^{\prime} \gamma_{0}$ en fonction du degré de pureté du matériau (le paramètre variable est $\left.\xi_{0} / l\right)$.

En supposant que l'on n'est pas au voisinage de la transition et que la fréquence est suffisamment faible (mais néanmoins supérieure à la fréquence de coupure) on peut écrire :

$$
\mu \omega \sigma \frac{\xi_{0}}{\xi} \lambda_{L}^{2} \ll 1 \text { et } \mu \omega \sigma \frac{\xi_{0}}{l} \lambda_{L}^{2} \ll 1 .
$$

D'où

$\frac{\delta \gamma_{0}}{\delta^{\prime} \gamma_{0}} \simeq\left(\frac{\sqrt{3}}{2 \pi}\right)^{1 / 3}\left(\frac{\xi^{3}}{\lambda_{L}^{2} \xi_{0}}\right)^{1 / 6}\left(1+\frac{\sqrt{3}}{2 \pi} \rho_{0}\left(1-\log \rho_{0}\right)\right)$

avec

$$
\rho_{0} \simeq \frac{2}{\pi} \frac{1}{\xi}\left(\frac{4}{3 \pi} \xi_{0} \lambda_{L}^{2}\right)^{1 / 3} .
$$

Le graphique 3 montre les résultats obtenus dans le cas de l'aluminium $\left(\xi_{0} \simeq 1,6 \times 10^{-6} \mathrm{~m}\right.$, $\left.\lambda_{L}(0) \simeq 1,65 \times 10^{-8} \mathrm{~m}, T \simeq 1 \mathrm{~K}\right)$.

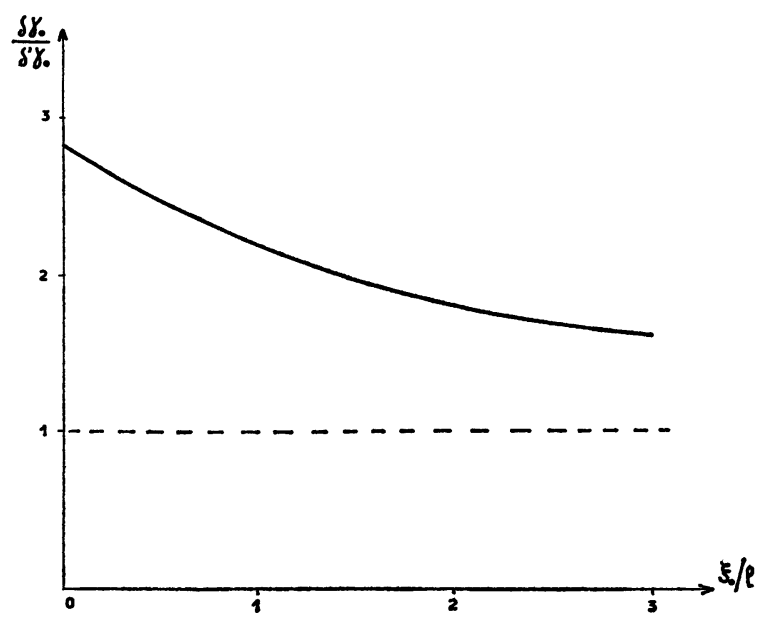

FIG. 3.

La courbe converge lentement vers la direction asymptotique correspondant à $\delta \gamma_{0} / \delta^{\prime} \gamma_{0}=1$ (matériau très impur).

On peut de même étudier le rapport $\alpha / \alpha^{\prime}$ en fonction 
de $\xi_{0} / l$. Le résultat est identique à celui obtenu dans une précédente étude sur les guides plans [1] (Fig. 4).

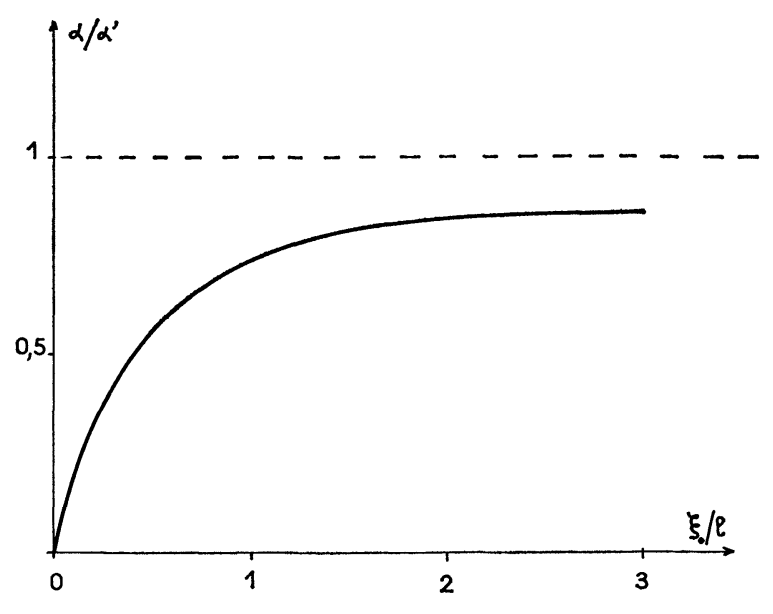

FIG. 4.

III. Profondeur de pénétration des champs.

1. Mode $\mathrm{TM}_{01}$. - La seule composante non nulle de $\mathbf{H}$ est $H_{\varphi}$.

La profondeur de pénétration à l'intérieur du matériau supraconducteur peut être définie par :

$$
\frac{1}{\lambda}=\operatorname{Re}\left(\frac{\left.H_{\varphi}\right|_{\rho=a}}{\int_{a}^{+\infty} H_{\varphi} d \rho}\right)
$$

Dans le cas où une formulation de type local est possible pour les densités de courants, on déduit, de l'expression des composantes des champs donnée précédemment dans l'étude de la propagation de l'onde et de l'équation :

$$
-i \mu \omega H_{\varphi}=\frac{\partial E_{\rho}}{\partial z}-\frac{\partial E_{z}}{\partial \rho} \quad \text { (équation de Maxwell) }
$$

la valeur de $H_{\varphi}$ :

$$
H_{\varphi}=\frac{1}{i \mu \omega}\left(1+\frac{\gamma^{2}}{\chi^{\prime 2}}\right) \frac{\partial E_{z}}{\partial \rho}
$$

ou compte tenu que l'on a dans les conditions habituelles $\left|\gamma^{2}\right| \ll \chi^{\prime 2}$ :

$$
H_{\varphi} \simeq \frac{1}{i \mu \omega} \frac{\partial E_{z}}{\partial \rho}
$$

On en déduit :

$$
\frac{1}{\lambda} \simeq \operatorname{Re}\left(i \mu \omega \frac{\left.H_{\varphi}\right|_{\rho=a}}{-\left.E_{z}\right|_{\rho=a}}\right) \simeq \operatorname{Re}\left(\frac{i \mu \omega}{Z_{S}}\right)
$$

où $Z_{S}$ est l'impédance de surface d'une surface plane. Dans le cadre d'une théorie locale (pour $\mathbf{J}_{N}$ et $\mathbf{J}_{S}$ ), on a :

$$
Z_{S}=i \mu \omega\left(\frac{1}{\lambda_{L}^{2}} \frac{\xi}{\xi_{0}}+i \mu \omega \sigma\right)^{-1 / 2}
$$

D'où :

$$
\frac{1}{\lambda}=\left(\left(\frac{1}{\lambda^{2}} \frac{\xi}{\xi_{0}}\right)^{2}+(\mu \omega \sigma)^{2}\right)^{1 / 4} \cos \frac{1}{2} \operatorname{Arctg}\left(\frac{\mu \omega \sigma \lambda_{L}^{2} \xi_{0}}{\xi}\right)
$$

avec $0<\operatorname{Arctg}<\pi / 2$.

On peut noter que pour une surface plane les amplitudes des champs seraient, dans le cas de la théorie locale, proportionnelles à $\mathrm{e}^{-x / \lambda}$ (l'axe ox étant normal à la surface).

En utilisant une théorie non locale la loi de variation de l'amplitude des champs n'est plus aussi simple mais on peut toujours écrire :

$$
\frac{1}{\lambda} \simeq \operatorname{Re}\left(\frac{i \mu \omega}{Z_{S}}\right)
$$

où $Z_{S}$ est ici l'impédance de surface déduite d'une formulation non locale (formule 6).

2. Mode $\mathrm{TE}_{01}$. - En utilisant les expressions des champs dans le cas où $\mathbf{J}_{N}$ et $\mathbf{J}_{S}$ sont décrits par des lois locales (matériau très impur) on a, à l'intérieur du matériau supraconducteur :

$$
\frac{H_{z}}{H_{\rho}}=\frac{\chi^{\prime} H_{0}^{(2)}\left(\chi^{\prime} \rho\right)}{i \gamma H_{1}^{(2)}\left(\chi^{\prime} \rho\right)}
$$

comme $\rho \geqslant a$ et compte tenu que $\left|\chi^{\prime} a\right| \gg 1$ on a :

$$
\frac{H_{z}}{H_{\rho}} \simeq-\frac{\chi^{\prime}}{\gamma}
$$

puisque $\left|\chi^{\prime}\right| \simeq|\gamma|$ on a également $\left|H_{\rho}\right| \ll\left|H_{z}\right|$.

Pratiquement le champ magnétique se réduit à sa composante $\mu H_{z}$. La profondeur de pénétration peut être définie par:

$$
\frac{1}{\lambda^{\prime}}=\operatorname{Re}\left(\frac{\left.H_{z}\right|_{\rho=a}}{\int_{a}^{+\infty} H_{z} d \rho}\right)
$$

En utilisant

$$
-i \mu \omega H_{z}=\frac{1}{\rho} \frac{\partial}{\partial \rho}\left(\rho E_{\varphi}\right)
$$

et la condition $\left|\chi^{\prime} \rho\right| \gg 1$.

On obtient :

$$
H_{z} \simeq-\frac{1}{i \mu \omega} \frac{i \chi^{\prime} \rho}{\left(1+i \chi^{\prime} \rho\right)} \frac{\partial E_{\varphi}^{*}}{\partial z} \simeq-\frac{1}{i \mu \omega} \frac{\partial E_{\varphi}}{\partial z} .
$$

D'où

$$
\frac{1}{\lambda^{\prime}} \simeq \operatorname{Re}\left(i \mu \omega \frac{\left.H_{z}\right|_{\rho=a}}{\left.E_{\varphi}\right|_{\rho=a}}\right) \simeq \operatorname{Re}\left(\frac{i \mu \omega}{Z_{S}}\right) .
$$

On retrouve la formule (23). Par conséquent $\lambda^{\prime}=\lambda$. Déterminons maintenant la valeur $\lambda$ commune aux modes $\mathrm{TE}_{01}$ et $\mathrm{TM}_{01}$ et correspondant à la théorie non locale (matériau pur). Utilisons (6) et étudions le cas plus particulier où $\frac{\mu \omega \sigma \xi_{0} \lambda_{L}^{2}}{l} \ll 1$. En négligeant 
des termes d'ordre supérieur, on obtient finalement [4] :

$$
\begin{aligned}
\lambda \simeq\left(\frac{\sqrt{3}}{2 \pi} \xi_{0} \lambda_{L}^{2}\right)^{1 / 3}\left\{1+\frac{\sqrt{3}}{\pi^{2}} \frac{1}{\xi}\left(\frac{4}{3 \pi} \xi_{0} \lambda_{L}^{2}\right)^{1 / 3} \times\right. \\
\times\left(1-\log \frac{2}{\pi} \frac{1}{\xi}\left(\frac{4}{3 \pi} \xi_{0} \lambda_{L}^{2}\right)^{1 / 3}\right) \\
\left.-\left(\frac{\mu \omega \sigma \xi_{0} \lambda_{L}^{2}}{3 l}\right)^{2}(\quad)\right\}
\end{aligned}
$$

$\operatorname{avec}()=$

$$
\begin{aligned}
1+\frac{\sqrt{3}}{\pi^{2}} \frac{1}{\xi}\left(\frac{4}{3 \pi} \xi_{0} \lambda_{L}^{2}\right)^{1 / 3}\left(-7+12 \frac{\xi}{l}-\frac{9}{2} \frac{\xi^{2}}{l^{2}}+\right. \\
\left.+\left(9 \frac{\xi}{l}-11\right) \log \frac{2}{\pi} \frac{1}{\xi}\left(\frac{4}{3 \pi} \xi_{0} \lambda_{L}^{2}\right)\right)^{1 / 3}
\end{aligned}
$$

En première approximation :

$$
\begin{gathered}
\lambda \simeq\left(\frac{\sqrt{3}}{2 \pi} \xi_{0} \lambda_{L}^{2}\right)^{1 / 3}\left\{1+\frac{\sqrt{3}}{\pi^{2}} \frac{1}{\xi}\left(\frac{4}{3 \pi} \xi_{0} \lambda_{L}^{2}\right)^{1 / 3} \times\right. \\
\left.\times\left(1-\log \frac{2}{\pi} \frac{1}{\xi}\left(\frac{4}{3 \pi} \xi_{0} \lambda_{L}^{2}\right)^{1 / 3}\right)\right\} .
\end{gathered}
$$

Pour un matériau très pur $\xi \simeq \xi_{0}$, on a dans ce cas :

$$
\begin{aligned}
\lambda \simeq\left(\frac{\sqrt{3}}{2 \pi} \xi_{0} \lambda_{L}^{2}\right)^{1 / 3} & \left\{1+\frac{\sqrt{3}}{\pi^{2}}\left(\frac{4}{3 \pi} \frac{\lambda_{L}^{2}}{\xi_{0}^{2}}\right)^{1 / 3} \times\right. \\
& \left.\times\left(1-\log \frac{2}{\pi}\left(\frac{4}{3 \pi} \frac{\lambda_{L}^{2}}{\dot{\zeta}_{0}^{2}}\right)^{1 / 3}\right)\right\}
\end{aligned}
$$

L'expression précédente permet d'évaluer la correction à apporter à la valeur limite de Pippard :

$$
\left(\frac{\sqrt{3}}{2 \pi} \xi_{0} \lambda_{L}^{2}\right)^{1 / 3}
$$

Dans le cas de l'aluminium la valeur de $\lambda$ calculée d'après (27) pour $T=0\left(H \ll H_{\mathrm{c}}\right)$ est $\left(\xi_{0}=16000 \AA\right.$, $\left.\lambda_{L}(0) \simeq 165 \AA\right\} \lambda=515 \AA$ en accord avec les valeurs expérimentales (extrapolées de valeurs à $T>0$ ) comprises entre $490 \AA$ et $515 \AA$.

De même pour l'étain on aurait $\left(\xi_{0}=2400 \AA\right.$, $\left.\lambda_{L}(0)=365 \AA\right) \lambda \simeq 500 \AA$, les valeurs expérimentales étant comprises entre $500 \AA$ et $510 \AA$.

En présence d'impuretés, on peut utiliser (26) à condition que le degré d'impureté ne soit pas trop grand car alors les conditions de validité de (26) ne seraient plus satisfaites. En effet (26) se déduit de (6) qui n'est valable que si $\left|g_{2}\right| /\left|g_{1}^{4 / 3}\right| \ll 1$.

Conclusion. - En résumé on voit que le formalisme utilisé ici permet, tout en rendant compte des réalités expérimentales, d'obtenir des résultats analytiques pour les diverses grandeurs caractérisant la propagation guidée. Ceci permet d'éviter par conséquent d'avoir recours aux méthodes du calcul numérique nécessaires en pratique dans d'autres formalismes. Les résultats que nous obtenons peuvent être exprimés d'une façon relativement simple en fonction des différents paramètres usuels caractérisant le supraconducteur, tout en tenant compte de la présence éventuelle d'impuretés dans le matériau utilisé.

\section{Bibliographie}

[1] Zepp (G.), J. Physique, 1970, 31, 513.

[2] Biondi (M. A.), Garfunkel (M. P.), Phys. Rev., 1959, 116, 853.

[3] Zepp (G.), C. R. Acad. Sci., Paris, 1970, 270B, 30.

[4] Zepp (G.), C. R. Acad. Sci., Paris, 1970, 270B, 785.
[5] Abrikosov (A. A.), Gor'kov (L. P.), Khalatnikov (I. M.), Soviet Physics J. E. T. P., 1959, 35, 182.

[6] Mattis (D. C.), Bardeen (J.), Phys. Rev., 1958, 111, 412.

[7] Hahn (H.), Halama (H. J.), Brookhaven National Laboratory, 1968, rapport AADD. 